\title{
Burst-mode EDFA based on a mid-position gain flattening filter with an overpumping configuration for variable traffic conditions in a WDM environment
}

\author{
Yoshinari Awaji · Hideaki Furukawa - Naoya Wada • \\ Eddie Kong • Peter Chan · Ray Man
}

Received: 31 August 2007 / Accepted: 6 April 2008 / Published online: 24 April 2008

(C) The Author(s) 2008

\begin{abstract}
The WDM flatness easily collapses due to substantial changes in input power, an inherent problem in optical packet communication because of dynamic changes in traffic density. We propose a burst-mode EDFA with a novel configuration based on the concept of a mid-position gain flattening filter (GFF) with overpumping. This configuration can effectively suppress gain transience while maintaining WDM flatness.
\end{abstract}

Keywords Burst-mode EDFA - Transient response - Overpumping · Gain flattening filter · Traffic conditions

\section{Introduction}

It is well known that conventional erbium-doped fiber amplifiers (EDFAs) tend to show significant transient responses in the presence of bursty changes in the input signal, despite the suitability of EDFAs for amplification of high-bit-rate continuous signals. This is because of their low pattern effect compared with semiconductor amplifiers due to the relatively long lifetime of the $\mathrm{Er}^{3+}$ ions. Such bursty traffic is generated by adding/dropping in $\lambda$-switched networks, or in next-generation switching schemes such as optical packet switching (OPS) and optical burst switching (OBS). Automatic gain control (AGC) has been considered one of the most promising candidates to customize EDFAs for application in bursty traffic environments (Nakaji et al. 2003; Tian and Kinoshita 2003; Bianciotto et al. 2003). However, the response time of AGC is sub-microsecond at best (Nakaji et al. 2003; Tian and Kinoshita

Y. Awaji $(\bowtie) \cdot$ H. Furukawa $\cdot$ N. Wada

New Generation Network Research Center, National Institute of Information and Communications

Technology (NICT), 4-2-1, Nukuikita, Koganei, Tokyo 184-8795, Japan

e-mail: yossy@nict.go.jp

E. Kong · P. Chan · R. Man

Amonics Ltd., Unit 101, 1/F, Winning Centre, 29 Tai Yau Street, San Po Kong, Kowloon, Hong Kong,

People's Republic of China 
2003), which is too slow for the extremely short duration of typical optical packets (Wada et al. 2003).

We have previously showed that adoption of EDF with a larger active area successfully reduced transience for optical packets of $400 \mathrm{~ns}$ duration at both a single wavelength (Awaji et al. 2007a) and in a WDM environment (Awaji et al. 2007b,c). Our approach to realize an EDFA with inherently low transience can be termed a "static" approach because it does not need any additional devices to control the gain of the EDFA. On the other hand, AGC generally deployed to suppress transience needs an optical power monitor and an additional circuit to control the gain. We classify approaches involving gain control as "dynamic", in contrast with the static approach described above. In other words, the dynamic approach involves compensation of the transience, whereas the static approach involves mitigation. The static approach was shown to be quite effective for optical packets with short duration $(<400 \mathrm{~ns})$ because there is no bandwidth limitation. Moreover, the advantages of the static approach will increase in future, considering the trend towards higher payload bit rates, resulting in reduced packet duration.

Despite the superiority of the static approach over dynamic approaches, the issue of vulnerability to changes in input power in WDM environments still remains. Such changes in input power, which are easily caused by changes in traffic density of optical packets, deteriorate the gain flatness of WDM channels. This problem is not confined to the static approach, but occurs in most EDFAs. This deterioration of the gain flatness increases the variation of residual gain transience.

In this paper, we show an improved configuration based on the static approach. The new configuration was designed by numerical simulation and optimization in order to suppress collapse of gain flatness of the WDM channels, which accommodate optical packet traffic, caused by drastic changes in the input power. We used Optisystem v5.0 (Optiwave Systems Inc.) for simulation and design.

\section{Principle of transience and design strategy}

The principle of transience is approximately described by the following equations (Tian and Kinoshita 2003; Sun et al. 1999):

$$
\begin{aligned}
& G^{\prime}(0)=\frac{[G(\infty)-G(0)]}{\tau_{0}}\left[1+\sum_{j} \frac{P^{\mathrm{out}}\left(\lambda_{j}\right)}{P^{\mathrm{IS}}\left(\lambda_{j}\right)}\right], \\
& P^{\mathrm{IS}}\left(\lambda_{j}\right)=\frac{h v S}{\left[\sigma_{a}\left(\lambda_{j}\right)+\sigma_{e}\left(\lambda_{j}\right)\right] \Gamma_{j} \tau_{0}},
\end{aligned}
$$

where $G(0)$ is the gain before transience, $G(\infty)$ is the steady-state gain after transience, $\tau_{0}$ is the intrinsic lifetime of the upper level of the $\mathrm{Er}^{3+}$ ions, $P^{\text {out }}$ is the output power, $P^{\mathrm{IS}}$ is the intrinsic saturation power, $S$ is the active erbium area of the EDF, $\sigma_{a}$ and $\sigma_{e}$ are absorption and emission cross-sections, and $\Gamma$ is the confinement factor.

A major cause of transience is considered to be cross gain saturation, according to Eqs. (1) and (2). These equations are also implemented as a ready-made component named "EDF Dynamic" in the Optisystem software.

To suppress transience, in a previous study, we adopted an EDF with a larger $S$ having a diameter of $7.8 \mu \mathrm{m}$ (Awaji et al. 2007c) as a first example of the static approach. This approach was quite effective because the transience, which is described by the time derivative $G^{\prime}(0)$, 

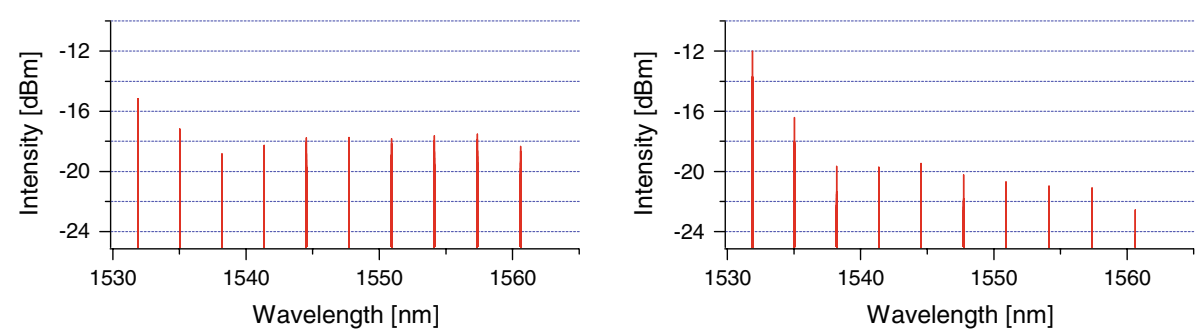

Fig. 1 Collapse of WDM flatness caused by substantial changes in input power to EDFA for ten 400-GHz spaced channels on ITU grid $(1531.90-1560.61 \mathrm{~nm})$. Left: $-2.1 \mathrm{dBm}$ input, Right: $-14.6 \mathrm{dBm}$ input

is approximately inversely proportional to $S$. Thus, the static approach can be considered to alleviate saturation. We used the same value of $S$ in the following simulations.

Here, we note the WDM flatness of a burst-mode EDFA. A conventional EDFA is designed to realize WDM flatness for an expected input power by using a gain flattening filter (GFF) or precise optimization of the length of the EDF and the pumping power. However, if the peak power of the packet is kept constant, the average power will change according to the traffic density. Actually, the flatness easily deteriorates when the input power substantially changes; an example of the simulated collapse of WDM flatness of an EDFA is shown in Fig. 1. The gain difference between channels increased to $10.5 \mathrm{~dB}$.

Such changes in the input power to an EDFA are quite usual in optical packet-based communications. Ironically, therefore, a burst-mode EDFA is actually vulnerable to substantial changes in input power according to the packet density. Such collapse of the gain flatness can be a problem not only for WDM transmission but also for suppression of transience. This is because variation of the output power $\left(P^{\text {out }}\right)$ with wavelength induces changes in the transience, as easily predicted from Eqs. (1) and (2). The transience is reliably suppressed by means of the static configuration, but residual transience can be affected by possible variations in the traffic. Therefore, we propose an improved static configuration, described below, to suppress the transience evenly in all wavelength channels under several conditions of traffic density, in other words, average power.

Overpumping is one possible scheme to reduce the collapse of the gain flatness caused by changes in input power. The overpumping scheme keeps $\mathrm{Er}^{3+}$ ions in nearly full inversion. Therefore, the sensitivity to changes in the input signal power should be reduced. It can also be considered as one means of alleviating saturation. However, the gain flatness may not be maintained under natural overpumping conditions. Therefore, it is necessary to adopt an overpumping scheme with a GFF.

In a conventional EDFA, the GFF is located after the EDF gain medium (so called postGFF) to achieve the best noise figure (NF). Although flatness is achieved after the GFF, it can be easily understood from Eqs. (1) and (2) that the degree of transience may vary according to the wavelength channels used. Traffic density and the timing of arriving packets on each wavelength channel are independent of each other; hence, the wavelength channel used continuously changes. The output power ( $\left.P^{\text {out }}\right)$ at the end of the EDF is governed by the spectrum of the emission cross-section, and the transience differs depending on $P^{\text {out }}$ just before the GFF. For example, we can easily conceive a case where arriving packets from an OPS network are tentatively distributed among available wavelength channels; therefore, the packets experience different transience according to the wavelength in the same EDFA. It should be noted that the static approach involving increasing the active erbium area can reliably reduce the transience but cannot neglect it completely. 


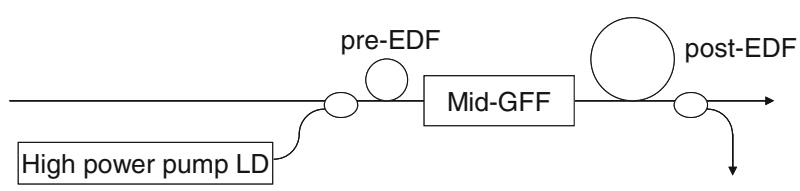

Fig. 2 Improved static configuration of EDFA with mid-GFF and overpumping

In view of the requirements for gain flatness, tolerance to changes in incident power, low transience, and lower NF, our novel proposal is to use a mid-positioned GFF (called mid-GFF) with overpumping configuration, as shown in Fig. 2. In contrast to post-GFF, a configuration in which the GFF is located before the EDF (pre-GFF) possibly reduces variations in transience depending on the wavelength, though it results in worse NF. The mid-GFF configuration shares both the merits and demerits of the pre- and post-GFF schemes.

The procedure adopted for designing the mid-GFF configuration was as follows.

1) The total length of EDF should be decided to maintain nearly full inversion regarding the available pumping power and dopant density.

2) Profiles of the GFF should be numerically calculated in order to achieve gain flatness, taking account of the expected gain spectrum given by the EDF. The gain spectrum is an accumulation of the spectra of the separate EDFs before and after the GFF (called preand post-EDFs). The balance of the lengths of pre- and post-EDFs should be adjusted to find the nearly optimum condition within the total length of the EDF. To do so, we used the WDM optimization function of the Optisystem software.

3) Appropriate balance of the pre- and post-EDFs should be selected concerning variations in transience, WDM flatness, and total NF, as shown in the next section.

\section{Results of simulation}

We assumed an optical packet payload length of 4000 bits at $10 \mathrm{Gbps}$ located on an ITU grid (1531.90-1560.61 nm) with $400 \mathrm{GHz}$ spacing. We compared two traffic densities $(\rho)$ at the optical layer, $33 \%$ and $0.37 \%$, which could be changed by adjusting the time period between each packet.

To reduce the calculation time, we treated only the envelope of the packets. The extinction ratio of the packet modulator was set to $23 \mathrm{~dB}$, the total length of EDF was set to $2.5 \mathrm{~m}$., and the average input powers for each traffic density were $-2.1 \mathrm{dBm}$ and $-14.6 \mathrm{dBm}$, respectively.

Figure 3 shows example GFF profiles used in the simulation when the balance of pre- and post-EDFs was changed in $50 \mathrm{~cm}$ steps. The ten vertical dotted lines represent the location of ten wavelength channels. It can be seen that the loss on the shortest wavelength $(1531.90 \mathrm{~nm})$ might become worse because of increased excess losses according to the change of balance between the pre- and post-EDFs. In particular, the loss, hence the NF, drastically worsened when the pre-EDF was longer than $150 \mathrm{~cm}$.

Figure 4 shows an example of simulated gain flatness of an EDFA with a mid-GFF configuration and overpumping, in comparison with Fig. 1. By means of overpumping, collapse of the gain flatness due to changes in input power was successfully suppressed compared with Fig. 1. The gain difference between channels remained within $4.3 \mathrm{~dB}$ at most.

Finally, we simulated variation of the NF and the degree of transience according to the balance of pre- and post-EDFs, as shown in Fig. 5. When all wavelengths are present, their contributions to $P^{\text {out }}$ are summed and averaged on every channel, thereby obscuring the 


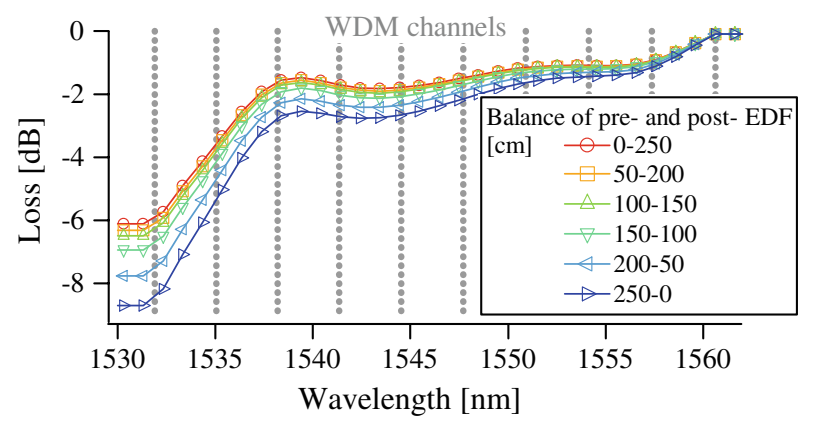

Fig. 3 Examples of GFF profiles. Dotted lines indicate 10 wavelength channels
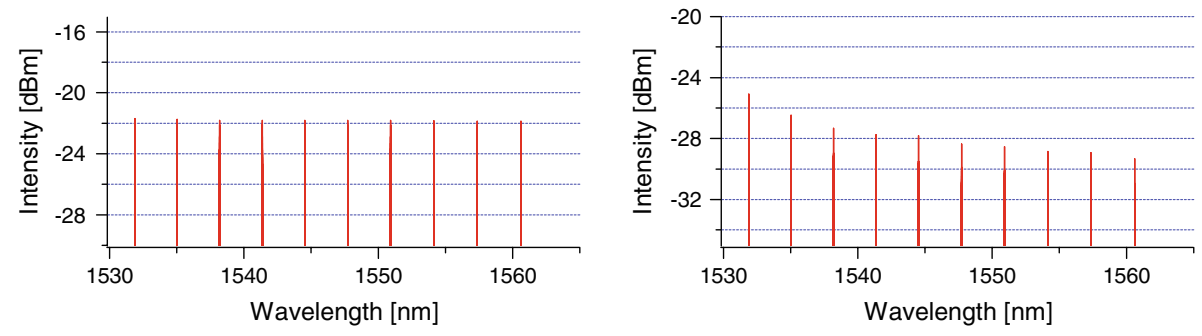

Fig. 4 WDM flatness maintained by mid-GFF with overpumping, configured as $1.5 \mathrm{~m}$ EDF + midGFF + 1.0m EDF. Left: $-2.1 \mathrm{dBm}$ input, $\rho=33 \%$; Right: $-14.6 \mathrm{dBm}$ input, $\rho=0.37 \%$

Degree of the transience $[\%]=(a-b) / a$

$$
\left(\begin{array}{l}
\text { Packet length : } 500 \text { byte } \\
=4000 \text { bit }(\sim 400 \mathrm{~ns} @ 10 \mathrm{Gbps})
\end{array}\right)^{\mathrm{a}}
$$
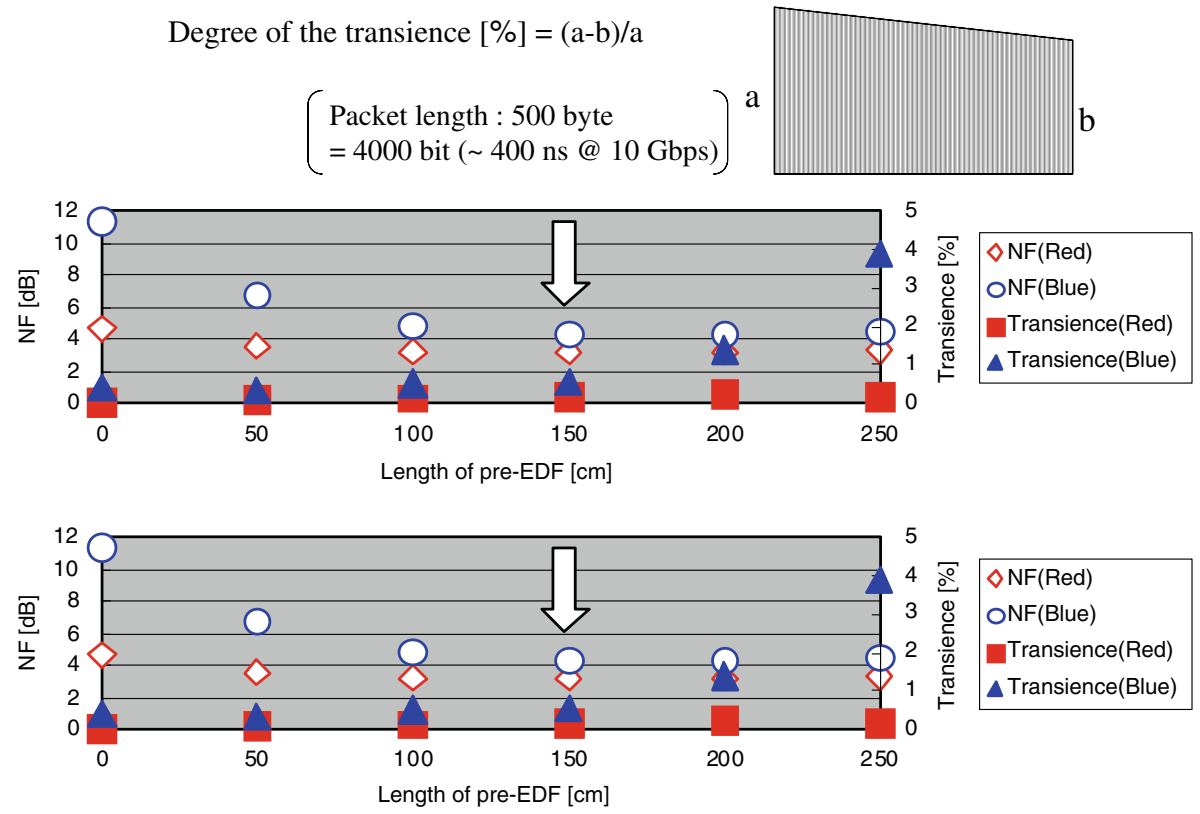

Fig. 5 Top: Definition of degree of transience. Middle, bottom: variation of NF and transience depending on balance of pre- and post-EDFs for different traffic densities (middle: $\rho=0.37 \%$, bottom: $\rho=33 \%$ ). Total EDF length $=250 \mathrm{~cm}$. Arrow indicates one compromise condition regarding trade-off between NF and transience for application to optical packet switching 
wavelength dependency of the transience. In order to clarify the wavelength dependence for a possibly existing packet, as representative channels, we chose two pairs of adjacent channels from both ends of the 10 available channels, called the red band and the blue band. Both channels in the blue and red bands were amplified, and one channel at each end $(1531.90 \mathrm{~nm}$ and $1560.61 \mathrm{~nm}$ as the blue and red channels) was observed. The NF was monitored using the "WDM analyzer" function of the Optisystem software, and the transience was measured from waveforms of the calculated packets. The degree of transience was defined as illustrated at the top of Fig. 5. In this example, the condition that the length of the pre-EDF be $150 \mathrm{~cm}$ is a compromise in terms of the NF and transience requirements for each channel, considering the application to OPS systems.

\section{Summary}

Vulnerability of burst-mode EDFAs to substantial changes in input power, resulting in collapse of the WDM gain flatness, is an inherent issue regardless of whether a static or dynamic approach is adopted to suppress the gain transience. Such substantial changes in input power are easily caused by changes in traffic density. We proposed a so-called mid-GFF with overpumping configuration as one promising design of burst-mode EDFAs for OPS applications to suppress collapse of the gain flatness, while maintaining low transience. From numerical simulations, we found a trade-off between the noise factor (NF) and transience. Therefore, it is necessary to select a compromise condition in view of the application. In this study, the $\mathrm{NF}$ was $<4.3 \mathrm{~dB}$ and the residual transience was less than $0.58 \%$ under the selected compromise condition. Moreover, the WDM flatness was improved by $6.2 \mathrm{~dB}$ compared with a conventional EDFA. Thus, we showed that the proposed improved static configuration can realize both mitigation of transience and WDM flatness in the presence of changes in input power.

Open Access This article is distributed under the terms of the Creative Commons Attribution Noncommercial License which permits any noncommercial use, distribution, and reproduction in any medium, provided the original author(s) and source are credited.

\section{References}

Awaji, Y., Furukawa, H., Wada, N., Chan, P., Man, R.: Mitigation of transient response of Erbium-doped fiber amplifier for burst traffic of high speed optical packets. In: Proc. CLEO 2007, JTuA133, May (2007a)

Awaji, Y., Furukawa, H., Wada, N., Kong, E., Chan, P., Man, R.: Impact of transient response of erbium-doped fiber amplifier for OPS/WDM and its mitigation. In: Proc. ONDM 2007, Tu2.5, May (2007b)

Awaji, Y., Furukawa, H., Wada, N., Kong, E., Chan, P., Man, R.: Burst-mode EDFA with extremely low gain transient for $0.037 \sim 32 \%$ range of traffic density of optical packet stream. In: OECC 2007, PDP2-4 $(2007 \mathrm{c})$

Bianciotto, A., Carena, A., Ferrero, V., Gaudino, R.: EDFA gain transients: experimental demonstration of a low cost electronic control. IEEE Photon Technol. Lett. 15(10), 1351-1353 (2003)

Nakaji, H., Nakai, Y., Shigematsu, M., Nishimura, M.: Superior high-speed automatic gain controlled erbiumdoped fiber amplifiers. Opt. Fiber Technol. 9, 25-35 (2003)

Sun, Y., Zyskind, J.L., Srivastava, A.K., Zhang, L.: Analytical formula for the transient response of erbiumdoped fiber amplifiers. Appl. Opt. 38(9), 1682-1685 (1999)

Tian, C., Kinoshita, S.: Analysis and control of transient dynamics of EDFA pumped by 1480- and 980-nm lasers. J. Lightwave Technol. 21(8), 1728-1734 (2003)

Wada, N., Harai, H., Kubota, F.: 40 Gbit/s interface, optical code based photonics packet switch prototype. Proc. OFC 2003, FS7, March (2003) 